\title{
DIAGONAL EQUIVALENCE TO MATRICES WITH PRESCRIBED ROW AND COLUMN SUMS. II
}

\author{
RICHARD SINKHORN
}

A BSTRACT. Let $A$ be a nonnegative $m \times n$ matrix and let $r=$ $\left(r_{1}, \cdots, r_{m}\right)$ and $c=\left(c_{1}, \cdots, c_{n}\right)$ be positive vectors such that $\Sigma_{i=1}^{m} r_{i}=\Sigma_{j=1}^{n} c_{j}$. It is well known that if there exists a nonnegative $m \times n$ matrix $B$ with the same zero pattern as $A$ having the $i$ th row sum $r_{i}$ and $j$ th column sum $c_{j}$, there exist diagonal matrices $D_{1}$ and $D_{2}$ with positive main diagonals such that $D_{1} A D_{2}$ has $i$ th row sum $r_{i}$ and $j$ th column sum $c_{j}$. However the known proofs are at best cumbersome. It is shown here that this result can be obtained by considering the minimum of a certain real-valued function of $n$ positive variables.

It has been shown originally by Sinkhorn and Knopp [8] and Brualdi, Parter, and Schneider [3] that if $A$ is a nonnegative fully indecomposable matrix, i.e. $A$ contains no $s \times(n-s)$ zero submatrix, then there exists a doubly stochastic matrix of the form $D_{1} A D_{2}$ where $D_{1}$ and $D_{2}$ are diagonal matrices with positive main diagonals. Later Djoković [4], and independently, London [5], proved the same theorem by considering the minimum of

$$
f(x)=\prod_{i=1}^{n}\left(\sum_{j=1}^{n} a_{i j} x_{j}\right) / \prod_{j=1}^{n} x_{j}
$$

for vectors $x=\left(x_{1}, \cdots, x_{n}\right)$ with positive coordinates.

In the meantime Menon [6] had obtained the following modification of this result.

Theorem 1. Let $A$ be a nonnegative $m \times n$ matrix and let $r=\left(r_{1}, \cdots\right.$, $\left.r_{m}\right)$ and $c=\left(c_{1}, \cdots, c_{n}\right)$ be positive vectors such that $\sum_{i=1}^{m} r_{i}=\sum_{j=1}^{n} c_{j}$. If there exists a nonnegative $m \times n$ matrix $B$ with the same zero pattern as $A$, i.e. $b_{i j}=0 \Leftrightarrow a_{i j}=0$, having ith row sum $r_{i}$ and $j$ th column sum $c_{j}$, then 1973.

Received by the editors September 17, 1973 and, in revised form, October 31, 15A51.

AMS (MOS) subject classifications (1970). Primary 15A15, 15A21; Secondary

Key words and phrases. Nonnegative matrix, diagonal equivalence, fully indecomposable matrix, zero pattern. 
there exist diagonal matrices $D_{1}$ and $D_{2}$ with positive main diagonals such that $D_{1} A D_{2}$ has ith row sum $r_{i}$ and $j$ th column sum $c_{j} \cdot$

Brualdi [2] showed that the existence of $B$ in Theorem 1 is equivalent to the conditions that

(1) $A[E \mid F)=0, A(E \mid F] \neq 0 \Rightarrow \Sigma_{i \in E^{r}} r_{i}<\Sigma_{j \in F} c_{j}$, and

(2) $A[E \mid F)=0, A(E \mid F]=0 \Rightarrow \Sigma_{i \in E} r_{i}=\Sigma_{j \in F}{ }^{c}$, if $\sum_{i=1}^{m}{ }^{r} i=\sum_{j=1}^{n} c_{j}$ holds.

The notation used has the following meaning. If $E$ is a proper nonvoid subset of $M=\{1, \cdots, m\}$ and $F$ is a proper nonvoid subset of $N=\{1, \cdots$, $n\}$, then $A[E \mid F)$ is that submatrix of $A$ obtained by deleting from $A$ those rows whose indices do not belong to $E$ and those columns whose indices belong to $F$. The rows and columns of this submatrix appear in the same order as in $A$ : rows are counted from top to bottom; columns are counted from left to right. $A(E \mid F]$ is that submatrix of $A$ obtained by deleting from $A$ those rows whose indices belong to $E$ and those columns whose indices do not belong to $F$, where, as before, the rows and columns of this submatrix appear in the same order as in $A$. Observe that the submatrix $A(E \mid F]$ is the same as the submatrix $A[M-E \mid N-F)$. In the course of the paper two other submatrix notations are used. $A[E \mid F]$ is used to denote the submatrix $A[E \mid N-F)=A(M-E \mid F]$ in $A ; A(E \mid F)$ is used to denote the submatrix $A[M-E \mid F)=A(E \mid N-F]$ in $A$.

Menon and Schneider [7] have given another proof of the Menon-Brualdi results.

It is the intent of this paper to show how the Djokovic-London formula can be modified to yield the Menon-Brualdi-Schneider results.

We shall require the following lemma which follows at once from the concavity of the logarithm function. See $[1, \mathrm{p} .7]$.

Lemma. Let $x_{1}, \cdots, x_{n}, \lambda_{1}, \cdots, \lambda_{n}$ be nonnegative real numbers and put $\lambda_{1}+\cdots+\lambda_{n}=\lambda$. Then if $0^{0}$ is taken to be 1 ,

$$
\left(\sum_{k=1}^{n} \lambda_{k} x_{k}\right)^{\lambda} \geq \lambda\left(\prod_{k=1}^{n} x_{k}^{\lambda_{k}}\right) .
$$

We now prove the intended result. We shall assume that whenever there is a submatrix $A[E \mid F)=0$ in $A, A(E \mid F] \neq 0$, for otherwise we could establish the result for the submatrices $A[E \mid F]$ and $A(E \mid F)$. We assume that Brualdi's condition (1) holds and that $\sum_{i=1}^{m} r_{i}=\sum_{j=1}^{n} c_{j}$.

Put 


$$
\phi(x)=\prod_{i=1}^{m}\left(\sum_{j=1}^{n} a_{i j} x_{j}\right)^{r_{i}} / \prod_{j=1}^{n} x_{j}^{c_{j}}
$$

where $x=\left(x_{1}, \cdots, x_{n}\right)$ is positive, i.e. $x \in\left(R^{n}\right)^{+}$. We shall consider the problem of determining the minimum of $\phi$ on $\left(R^{n}\right)^{+}$. Since $\sum_{i=1}^{m}{ }^{r}{ }_{i}=\sum_{j=1}^{n} c_{j}$, $\phi(\lambda x)=\phi(x)$ for all $\lambda>0$ and thus we can restrict our attention to the set $K$ of $x \in\left(R^{n}\right)^{+}$for which $\|x\|=\left(x_{1}^{2}+\cdots+x_{n}^{2}\right)^{1 / 2}=1$.

Suppose on $K, x \rightarrow \Delta$, the boundary of $\left(R^{n}\right)^{+}$. Let $F=\left\{j \mid x_{j} \rightarrow 0\right\}$ and then set $E=\left\{i \mid a_{i j}=0\right.$ for all $\left.j \notin F\right\}$. Since $x \rightarrow \Delta$ on $K, F$ is a nonvoid proper subset of $\{1, \cdots, n\}$. Since every $c_{j}>0, E$ is a proper subset of $\{1, \cdots, m\}$. If $E=\varnothing, \phi(x) \rightarrow \infty$ as $x \rightarrow \Delta$. If $E \neq \varnothing$, we write $\phi(x)=$ $\phi_{1}(x) \phi_{2}(x)$ where

$$
\phi_{1}(x)=\prod_{i \in E}\left(\sum_{j=1}^{n} a_{i j} x_{j}\right)^{r_{i}} / \prod_{j \in F} x_{j}^{c_{j}}=\prod_{i \in E}\left(\sum_{j \in F} a_{i j} x_{j}\right)^{r_{i}} / \prod_{j \in F} x_{j}^{c_{j}}
$$

and

$$
\phi_{2}(x)=\prod_{i \notin E}\left(\sum_{j=1}^{n} a_{i j} x_{j}\right)^{r_{i}} / \prod_{j \notin F} x_{j}^{c_{j}}
$$

Since $\phi_{2}$ has a positive limit as $x \rightarrow \Delta$, we concentrate on $\phi_{1}$.

Let $B$ be as in Theorem 1. Then $B[E \mid F)=0$ and therefore $\Sigma_{j \in F} b_{i j}=$ $r_{i}$ for each $i \in E$ and hence from the Lemma

$$
\left(\sum_{j \in F} a_{i j} x_{j}\right)^{r_{i}} \geq r_{i}^{r_{i}} \frac{\Pi_{j \in F} a_{i j}^{b_{i j}}}{\Pi_{j \in F} b_{i j}^{b_{i j}}} \prod_{j \in F} x_{j}^{b_{i j}}=\theta_{i} \prod_{j \in F} x_{j}^{b_{i j}}
$$

for all $i \in E$, where $0^{0}$ is taken to be 1 . Whence

$$
\phi_{1}(x) \geq \prod_{i \in E} \theta_{i} / \prod_{j \in F} x_{j}^{c_{j}-\Sigma_{i \in E} b_{i j}} .
$$

Since $\sum_{i=1}^{m} b_{i j}=c_{j}, j=1, \cdots, n$, certainly $\Sigma_{i \in E} b_{i j} \leq c_{j}$ for every $j \in F$. However since $\Sigma_{j \in F} c_{j}>\Sigma_{i \in E} r_{i}=\Sigma_{i \in E} \Sigma_{j \in F} b_{i j}, \Sigma_{i \in E} b_{i j_{0}}<c_{j_{0}}$ for at least one $j_{0} \in F$. Thus $\phi_{1}(x) \rightarrow \infty$ and so $\phi(x) \rightarrow \infty$ as $x \rightarrow \Delta$.

It follows that $\phi$ achieves a minimum on $\left(R^{n}\right)^{+}$. At such a point $\bar{x}=$ $\left(\bar{x}_{1}, \cdots, \bar{x}_{n}\right), \partial \ln \phi(x) / \partial x_{k}=0$ for $k=1, \cdots, n$. Whence

$$
\sum_{i=1}^{m} r_{i}\left(a_{i k} / \sum_{j=1}^{n} a_{i j} \bar{x}_{j}\right)-c_{k} / \bar{x}_{k}=0
$$


$k=1, \cdots, n$. Put $\bar{y}_{i}=r_{i} / \sum_{j=1}^{n} a_{i j} \bar{x}_{j}, i=1, \cdots, m$, and then set $D_{1}=$ $\operatorname{diag}\left(\bar{y}_{1}, \cdots, \bar{y}_{m}\right), D_{2}=\operatorname{diag}\left(\bar{x}_{1}, \cdots, \bar{x}_{n}\right)$. Then $D_{1} A D_{2}$ satisfies the conclusion of Theorem 1 .

\section{REFERENCES}

1. E. F. Beckenbach and R. Bellman, Inequalities, 2nd rev. ed., Ergebnisse der Math. und ihrer Grenzgebiete, Heft 30, Springer-Verlag, New York, 1965. MR 33 \#236.

2. R. A. Brualdi, Convex sets of nonnegative matrices, Canad. J. Math. 20 (1968), 144-157. MR 36 \#2636.

3. R. A. Brualdi, S. V. Parter and H. Schneider, The diagonal equivalence of a nonnegative matrix to a stochastic matrix, J. Math. Anal. Appl. 16 (1966), 31-50. MR 34 \#5844.

4. D. Z. Djoković, Note on nonnegative matrices, Proc. Amer. Math. Soc. 25 (1970), 80-82. MR 41 \#1768.

5. D. London, On matrices with a doubly stochastic pattern, J. Math. Anal. Appl. 34 (1971), 648-652. MR 43 \#7448.

6. M. V. Menon, Matrix links, an extremization problem, and the reduction of a nonnegative matrix to one with prescribed row and column sums, Canad. J. Math. 20 (1968), 225-232. MR $36 \# 3804$.

7. M. V. Menon and H. Schneider, The spectrum of a nonlinear operator associated with a matrix, Linear Algebra and Appl. 2 (1969), 321-334. MR 40 \#162.

8. R. Sinkhorn and P. Knopp, Concerning nonnegative matrices and doubly stochastic matrices, Pacific J. Math. 21 (1967), 343-348. MR 35 \#1617.

DE PARTMENT OF MATHEMATICS, UNIVERSITY OF HOUSTON, HOUSTON, TEXAS 77004 